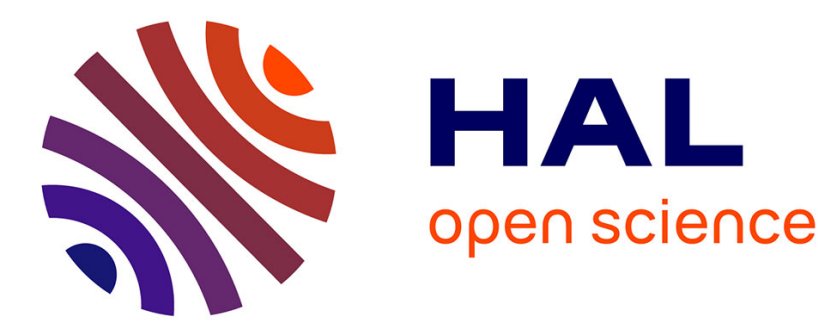

\title{
Residual Finite Tree Automata
}

Julien Carme, Rémi Gilleron, Marc Tommasi, Alain Terlutte, Aurélien Lemay

\section{To cite this version:}

Julien Carme, Rémi Gilleron, Marc Tommasi, Alain Terlutte, Aurélien Lemay. Residual Finite Tree Automata. 7th International Conference on Developments in Language Theory, Jul 2003, Szeged, Hungary, Hungary. pp.171 - 182. inria-00091272v2

HAL Id: inria-00091272

https://hal.inria.fr/inria-00091272v2

Submitted on 5 Sep 2006

HAL is a multi-disciplinary open access archive for the deposit and dissemination of scientific research documents, whether they are published or not. The documents may come from teaching and research institutions in France or abroad, or from public or private research centers.
L'archive ouverte pluridisciplinaire HAL, est destinée au dépôt et à la diffusion de documents scientifiques de niveau recherche, publiés ou non, émanant des établissements d'enseignement et de recherche français ou étrangers, des laboratoires publics ou privés. 


\title{
Residual Finite Tree Automata ${ }^{\star}$
}

\author{
J. Carme, R. Gilleron, A. Lemay, A. Terlutte, and M. Tommasi \\ Grappa - EA 3588 - Lille 3 University \\ http://www.grappa.univ-lille3.fr
}

\begin{abstract}
Tree automata based algorithms are essential in many fields in computer science such as verification, specification, program analysis. They become also essential for databases with the development of standards such as XML. In this paper, we define new classes of non deterministic tree automata, namely residual finite tree automata (RFTA). In the bottom-up case, we obtain a new characterization of regular tree languages. In the top-down case, we obtain a subclass of regular tree languages which contains the class of languages recognized by deterministic top-down tree automata. RFTA also come with the property of existence of canonical non deterministic tree automata.
\end{abstract}

\section{Introduction}

The study of tree automata has a long history in computer science; see the survey of Thatcher [Tha73, and the texts of F. Gécseg and M. Steinby [GS84, GS96], and of the TATA group [CDG $\left.{ }^{+97}\right]$. With the advent of tree-based metalanguages (SGML and XML) for document grammars, new developments on tree automata formalisms and tree automata based algorithms have been done MLM01, Nev02]. Also, because of the tree structure of documents, learning algorithms for tree languages have been defined for the tasks of information extraction and information retrieval EFr02,GK02, LPH00]. We are currently involved in a research project dealing with information extraction systems from semi-structured data. One objective is the definition of classes of tree automata satisfying two properties: there are efficient algorithms for membership and matching, and there are efficient learning algorithms for the corresponding classes of tree languages.

In the present paper, we only consider finite ranked trees. There are bottomup (also known as frontier to root) tree automata and top-down (also known as root to frontier) tree automata. The top-down version is particularly relevant for some implementations because important properties such as membership ${ }^{1}$ can be solved without handling the whole input tree into memory. There are also deterministic tree automata and non-deterministic tree automata. Determinism is important to reach efficiency for membership and other decision properties. It is known that non-deterministic top-down, non-deterministic bottom-up, and deterministic bottom-up tree automata are equally expressive and define regular tree languages. But there is a tradeoff between efficiency and expressiveness because some regular (and even finite) tree languages are not recognized

\footnotetext{
* This research was partially supported by "TACT-TIC" région Nord-Pas-de-Calais - FEDER and the MOSTRARE INRIA project

${ }^{1}$ given a tree automaton $A$, decide whether an input tree is accepted by $A$.
} 
by deterministic top-down tree automata. Moreover, the size of a deterministic bottom-up tree automaton can be exponentially larger than the size of a non-deterministic one recognizing the same tree language. This drawback can be dramatic when the purpose is to build tree automata. This is for instance the case in the problem of tree pattern matching and in machine learning problems like grammatical inference.

The process of learning finite state machines from data is referred as grammatical inference. The first theoretical foundations were given by Gold Gol67 and first applications were designed in the field of pattern recognition. Grammatical inference mostly focused on learning string languages but recent works are concerned with learning tree languages [Sak90, Fer02, GK02]. In most works, the target tree language is represented by a deterministic bottom-up tree automaton. This is problematic because the time complexity of the learning algorithm depends on the size of the target automaton. Therefore, again it is crucial to define learning algorithms for non-deterministic tree automata. The reader should note that tree patterns GK02 satisfy this property.

Therefore the aim of this article is to define non-deterministic tree automata corresponding to sufficiently expressive classes of tree languages and having nice properties from the algorithmic viewpoint and from the grammatical inference viewpoint. For this aim, we extend previous works from the string case [DLT02a to the tree case and we define residual finite state automata (RFTA). The reader should note that learning algorithms for residual finite string automata have been defined [DLT01, DLT02b].

In Section 3, we study the bottom-up case. We define the residual language of a language $L$ w.r.t a ground term $t$ as the set of contexts $c$ such that $c[t]$ is a term in $L$. We define bottom-up residual tree automata as automata whose states correspond to residual languages. Bottom-up residual tree automata are non-deterministic and recognize regular tree languages. We prove that every regular tree language is recognized by a unique canonical bottom-up residual tree automaton, minimal according to the number of states. We give an example of regular tree languages for which the size of the deterministic bottom-up tree automata grows exponentially with respect to the size of the canonical bottomup residual tree automata.

In Section 4, we study the top-down case. We define the residual language of a language $L$ w.r.t a context $c$ as the set of ground terms $t$ such that $c[t]$ is a term in $L$. We define top-down residual tree automata as automata whose states correspond to residual languages. Top-down residual tree automata are non-deterministic tree automata. Interestingly, the class of languages recognized by top-down residual tree automata is strictly included in the class of regular tree languages and strictly contains the class of languages recognized by deterministic top-down tree automata. We also prove that every tree language in this family is recognized by a unique canonical top-down residual tree automaton; this automaton is minimal according to the number of states.

The definition of residual finite state automata comes with new decision problems. All of them rely on properties of residual languages. It is proved that all residual languages of a given tree language $L$ can be built in both top-down and bottom-up cases. From these constructions we obtain positive answers to decision problems like 'decide whether an automaton is a (canonical) RFTA'. 
The exact complexity bounds are not given but we conjecture that are identical than in the string case.

The present work is connected with the paper by Nivat and Podelski NP97. They consider a monoid framework, whose elements are called pointed trees (contexts in our terminology, special trees in Tho84]), to define tree automata. They define a Nerode congruence in the bottom-up case and in the top-down case. Their work leads to the generalization of the notion of deterministic to l-r-deterministic (context-deterministic in our terminology) for top-down tree automata. They have a minimization procedure for this class of automata. It should be noted that the class of languages recognized by context-deterministic tree automata (also called homogeneous tree languages) is strictly included in the class of languages recognized by residual top-down tree automata.

\section{Preliminaries}

We assume that the reader is familiar with basic knowledge about tree automata. We follow the notations defined in TATA $\left[\mathrm{CDG}^{+} 97\right]$.

A ranked alphabet is a couple $(\mathcal{F}$, Arity) where $\mathcal{F}$ is a finite set and Arity is a mapping from $\mathcal{F}$ into $\mathbb{N}$. The set of symbols of arity $p$ is denoted by $\mathcal{F}_{p}$. Elements of arity $0,1, \ldots p$ are respectively called constants, unary, $\ldots, p$-ary symbols. We assume that $\mathcal{F}$ contains at least one constant. In the examples, we use parenthesis and commas for a short declaration of symbols with arity. For instance, $a$ is a constant and $f($,$) is a short declaration for a binary symbol f$. The set of terms over $\mathcal{F}$ is denoted by $\mathcal{T}(\mathcal{F})$. Let $\diamond$ be a special constant which is not in $\mathcal{F}$. The set of contexts (also known as pointed trees in NP97 and special trees in Tho84), denoted by $\mathcal{C}(\mathcal{F})$, is the set of terms which contains exactly one occurrence of $\diamond$. The expression $c[\diamond]$ denotes a context, we only write $c$ when there is no ambiguity. We denote by $c[t]$ the term obtained from $c[\diamond]$ by replacing $\diamond$ by a term $t$.

A bottom-up Finite Tree Automaton ( $\uparrow-F T A)$ over $\mathcal{F}$ is a tuple $A=\left(Q, \mathcal{F}, Q_{f}, \Delta\right)$ where $Q$ is a finite set of states, $Q_{f} \subseteq Q$ is a set of final states, and $\Delta$ is a set of transition rules of the form $f\left(q_{1}, \ldots, q_{n}\right) \rightarrow q$ where $n \geq 0, f \in \mathcal{F}_{n}$, $q, q_{1}, \ldots, q_{n} \in Q$. In this paper, the size of an automaton refers to its size in number of states, so two automaton which have the same number of states but different number of rules are considered as having the same size. When $n=0 \mathrm{a}$ rule is written $a \rightarrow q$, where $a$ is a constant. The move relation is written $\rightarrow_{A}$ and $\rightarrow_{A}^{*}$ is the reflexive and transitive closure of $\rightarrow_{A}$. A term $t$ reaches a state $q$ if and only if $t \rightarrow_{A}^{*} q$. A state $q$ accepts a context $c$ if and only if there exists a $q_{f} \in Q_{f}$ such that $c[q] \rightarrow_{A}^{*} q_{f}$. The automaton $A$ recognizes a term $t$ if and only if there exists a $q_{f} \in Q_{f}$ such that $t \rightarrow{ }_{A}^{*} q_{f}$. The language recognized by $A$ is the set of all terms recognized by $A$, and is denoted by $L(A)$.

Two $\uparrow$-FTA are equivalent if they recognize the same tree language. A $\uparrow$ FTA $A=\left(Q, \mathcal{F}, Q_{f}, \Delta\right)$ is trimmed if and only if all its states can be reached by at least one term and accepts at least one context. A $\uparrow$-FTA is deterministic ( $\uparrow$-DFTA $)$ if and only if there are no two rules with the same left-hand side in its set of rules. A tree language is regular if and only if it is recognized by a bottom-up tree automaton. As any $\uparrow$-FTA can be changed into an equivalent 
trimmed $\uparrow$-DFTA, any regular tree language can be recognized by a trimmed $\uparrow$-DFTA.

Let $L$ be a tree language over a ranked alphabet $\mathcal{F}$ and $t$ a term. The bottomup residual language of $L$ relative to a term $t$, denoted by $t^{-1} L$, is the set of all contexts in $\mathcal{C}(\mathcal{F})$ such that $c[t] \in L$ :

$$
t^{-1} L=\{c \in \mathcal{C}(\mathcal{F}) \mid c[t] \in L\} .
$$

Note that a bottom-up residual language is a set of contexts, and not a tree language. The Myhill-Nerode congruence for tree languages can be defined by two terms $t$ and $t^{\prime}$ are equivalent if they define the same residual languages. From the Myhill-Nerode theorem fro tree languages, we get the following result: a tree language is recognizable if and only if the number of residual languages is finite.

A top-down finite tree automaton $(\downarrow$-FTA) over $\mathcal{F}$ is a tuple $\mathcal{A}=(Q, \mathcal{F}, I, \Delta)$ where $Q$ is a set of states, $I \subseteq Q$ is a set of initial states, and $\Delta$ is a set of rewrite rules of the form $q(f) \rightarrow f\left(q_{1}, \ldots, q_{n}\right)$ where $n \geq 0, f \in \mathcal{F}_{n}, q, q_{1}, \ldots, q_{n} \in Q$. Again, if $n=0$ the rule is written $q(a) \rightarrow a$. The move relation is written $\rightarrow_{A}$ and $\rightarrow_{A}^{*}$ is the reflexive and transitive closure of $\rightarrow_{A}$. A state $q$ accepts a term $t$ if and only if $q(t) \rightarrow{ }_{A}^{*} t$. A recognizes a term $t$ if and only if at least one of its initial states accepts it. The language recognized by $A$ is the set of all ground terms recognized by $A$ and is denoted by $L(A)$.

Any regular tree language can be recognized by a $\downarrow$-FTA. This means that $\downarrow$-FTA and $\uparrow$-FTA have the same expressive power. A $\downarrow$-FTA is deterministic ( $\downarrow$-DFTA $)$ if and only if its set of rules does not contain two rules with the same left-hand side. Unlike $\uparrow$-DFTA, $\downarrow$-DFTA are not able to recognize all regular tree languages.

Let $L$ be a tree language over a ranked alphabet $\mathcal{F}$, and $c$ a context of $\mathcal{C}(\mathcal{F})$. The top-down residual language of $L$ relative to $c$, denoted by $c^{-1} L$, is the set of ground terms $t$ such that $c[t] \in L$ :

$$
c^{-1} L=\{t \in \mathcal{T}(\mathcal{F}) \mid c[t] \in L\} .
$$

The definition of top-down residual languages comes with an equivalence relation on contexts. It is worth noting that it does not define a congruence over terms. Nonetheless, based on [NP97, it can be shown that a tree language $L$ is regular if and only if the number of top-down residual languages associated with $L$ is finite. In the proof, it is used that the number top-down residual languages is lower than the number of bottom-up residual languages.

\section{Bottom-up residual finite tree automata}

In this section, we introduce a new class of bottom-up finite tree automata, called bottom-up residual finite tree automata ( $\uparrow$-RFTA). This class of automata shares some interesting properties with both bottom-up deterministic and nondeterministic finite tree automata which both recognize the class of regular tree languages.

On the one hand, as $\uparrow$-DFTA, $\uparrow$-RFTA admits a unique canonical form, based on a correspondence between states and residual languages, whereas $\uparrow$-FTA does 
not. On the other hand, $\uparrow$-RFTA are non-deterministic and can be much smaller in their canonical form than their deterministic counter-parts.

\subsection{Definition and expressive power of bottom-up residual finite tree automata}

First, let us precise the nature of this correspondence, then let us give the formal definition of $\uparrow$-residual tree automata and describe their properties.

In order to establish the nature of this correspondence between states and residual languages, let us introduce the notion of state languages. The state language $C_{q}$ of a state $q$ is the set of contexts accepted by the state $q$ :

$$
C_{q}=\left\{c \in \mathcal{C}(\mathcal{F}) \mid \exists q_{f} \in Q_{f}, c[q] \rightarrow_{A}^{*} q_{f}\right\} .
$$

As shown by the following example, state languages are generally not residual languages:

Example 1. Consider the tree language $L=\left\{f\left(a_{1}, b_{1}\right), f\left(a_{1}, b_{2}\right), f\left(a_{2}, b_{2}\right)\right\}$ over $\mathcal{F}=\left\{f(),, a_{1}, b_{1}, a_{2}, b_{2}\right\}$. This language $L$ is recognized by the tree automaton $A=\left(\left\{q_{1}, q_{2}, q_{3}, q_{4}, q_{5}\right\}, \mathcal{F},\left\{q_{5}\right\}, \Delta\right)$ where $\Delta=\left\{a_{1} \rightarrow q_{1}, b_{1} \rightarrow q_{2}, b_{2} \rightarrow\right.$ $\left.q_{3}, a_{2} \rightarrow q_{4}, a_{1} \rightarrow q_{4}, f\left(q_{1}, q_{2}\right) \rightarrow q_{5}, f\left(q_{4}, q_{3}\right) \rightarrow q_{5}\right\}$. Residual languages of $L$ are $a_{1}^{-1} L=\left\{f\left(\diamond, b_{1}\right), f\left(\diamond, b_{2}\right)\right\}, b_{1}^{-1} L=\left\{f\left(a_{1}, \diamond\right)\right\}, b_{2}^{-1} L=\left\{f\left(a_{1}, \diamond\right), f\left(a_{2}, \diamond\right)\right\}$, $a_{2}^{-1} L=\left\{f\left(\diamond, b_{2}\right)\right\}, f\left(a_{1}, b_{1}\right)^{-1} L=\{\diamond\}$. The state language of $q_{1}$ is $\left\{f\left(\diamond, b_{1}\right)\right\}$, which is not a residual language. The tree $a_{1}$ reaches $q_{1}$, so each context accepted by $q_{1}$ is an element of the residual language $a_{1}^{-1} L$, which means that $C_{q_{1}} \subset a_{1}^{-1} L$. But the reverse inclusion is not true because $f\left(\diamond, b_{2}\right)$ is not an element of $C_{q_{1}}$. The reader should note that this situation is possible because $A$ is non-deterministic.

In fact, it can be proved (the proof is omitted) that residual languages are unions of state languages. For any $L$ recognized by a tree automaton $A$, we have

$$
\forall t \in T(\mathcal{F}), t^{-1} L=\bigcup_{q \in Q, t \rightarrow_{A}^{*} q} C_{q} .
$$

As a consequence, if $A$ is deterministic and trimmed, each residual language is a state language and conversely.

We can define a new class of non-deterministic automata stating that each state language must correspond to a residual tree language. We have seen that residual tree languages are related to the Myhill-Nerode congruence and we will show that minimization of tree automata can be extended in the definition of a canonical form for this class of non-deterministic tree automata.

Definition 1. A bottom-up residual tree automaton ( $\uparrow-R F T A)$ is a $\uparrow-F T A A=$ $\left(Q, \mathcal{F}, Q_{f}, \Delta\right)$ such that $\forall q \in Q, \exists t \in T(\mathcal{F}), C_{q}=t^{-1} L(A)$.

According to the above definition and previous remarks, it can be shown that every trimmed $\uparrow$-DFTA is a $\uparrow$-RFTA. As a consequence, $\uparrow$-RFTA have the same expressive power than finite tree automata: 
Theorem 1. The class of tree languages recognized by $\uparrow-R F T A$ is the class of regular tree languages.

As an advantage of $\uparrow$-RFTA, the number of states of an $\uparrow$-RFTA can be much smaller than the number of states of any equivalent $\uparrow$-DFTA:

Proposition 1. There exists a sequence $\left(L_{n}\right)$ of regular tree languages such that for each $L_{n}$, the size of the smallest $\uparrow-D F T A$ which recognizes $L_{n}$ is an exponential function of $n$, and the size of the smallest $\uparrow-R F T A$ which recognizes $L_{n}$ is a linear function of $n$.

Sketch of proof We give an example of regular tree languages for which the size of the $\uparrow$-DFTA grows exponentially with respect to the size of the equivalent canonical $\uparrow$-RFTA. A path is a sequence of symbols from the root to a leaf of a tree. The length of a path is the number of symbols on the path, except the root. Let $\mathcal{F}=\{f(), a$,$\} and let us consider the tree language L_{n}$ which contains exactly the trees with at least one path of length $n$. Let $A_{n}=\left(Q, \mathcal{F}, Q_{f}, \Delta\right)$ be a $\uparrow$-FTA defined by: $Q=\left\{q_{*}, q_{0}, \ldots, q_{n}\right\}, Q_{f}=\left\{q_{0}\right\}$ and

$$
\begin{gathered}
\Delta=\left\{a \rightarrow q_{*}, a \rightarrow q_{n}, f\left(q_{*}, q_{*}\right) \rightarrow q_{*}\right\} \cup \\
\bigcup_{k \in[1, \ldots, n], q \in Q \backslash\left\{q_{0}\right\}}^{n}\left\{f\left(q_{k}, q\right) \rightarrow q_{k-1}, f\left(q, q_{k}\right) \rightarrow q_{k-1}, f\left(q_{k}, q\right) \rightarrow q_{*}, f\left(q, q_{k}\right) \rightarrow q_{*}\right\}
\end{gathered}
$$

Let $C_{*}$ be the set of contexts which contain at least one path of length $n$. Let $C_{i}$ be the set of contexts whose path from the root to $\diamond$ is of length $i$. Let $t_{*}$ be a term such that all its paths are of length greater than $n$. Note that the set of contexts $c$ such that $c\left[t_{*}\right]$ belongs to $L_{n}$ is exactly the set of contexts $C_{*}$. Let $t_{0} \ldots t_{n}$ be terms such that for all $i \leq n, t_{i}$ contains exactly one path of length smaller than $n$, and the length of this path is $n-i$. Therefore, $t_{i}^{-1} L_{n}$ is the set of contexts $C_{*} \cup C_{i}$.

One can verify that $C_{q_{*}}$ is exactly $t_{*}^{-1} L_{n}=C_{*}$, and for all $i \leq n, C_{q_{i}}$ is exactly $t_{i}^{-1} L_{n}=C_{*} \cup C_{i}$. The reader should note that rules of the form $f\left(q_{k}, q\right) \rightarrow q_{*}$ and $f\left(q, q_{k}\right) \rightarrow q_{*}$ are not useful to recognize $L_{n}$ but they are required to obtain a $\uparrow$-RFTA (because $C_{i}$ is not a residual language of $L_{n}$ ). So $A_{n}$ is a $\uparrow$-RFTA and recognizes $L_{n}$. The size of $A_{n}$ is $n+2$.

The construction of the smallest $\uparrow$-DFTA which recognizes $L\left(A_{n}\right)$ is left to the reader. But, it can easily be shown that the number of states is in $O\left(2^{n}\right)$ because states must store lengths of all paths smaller than $n$.

Unfortunately, the size of a $\uparrow$-RFTA can be exponentially larger than the size of an equivalent $\uparrow$-FTA.

\subsection{The canonical form of bottom-up residual tree automata}

As $\uparrow$-DFTA, $\uparrow$-RFTA have the interesting property to admit a canonical form. In the case of $\uparrow$-DFTA, there is a one-to-one correspondence between residual languages and state languages. This is a consequence of the Myhill-Nerode theorem for trees.

A similar result holds for $\uparrow$-RFTA. In a canonical $\uparrow$-RFTA, the set of states is in one-to-one correspondence with a subset of residual languages called prime residual languages. 
Definition 2. Let $L$ be a tree language. A bottom-up residual language of $L$ is composite if and only if it is the union of the bottom-up residual languages that it strictly contains:

$$
t^{-1} L=\bigcup_{t^{\prime-1} L \subsetneq t^{-1} L} t^{\prime-1} L
$$

A residual language is prime if and only if it is not composite.

Example 2. Let us consider again the tree languages in the proof of Proposition 1. Let $Q_{n}$ be the set of states of $A_{n}$. All the $n+2$ states $q_{*}, q_{0}, \ldots, q_{n}$ of $Q_{n}$ have state languages which are prime residual languages. The subset construction applied on $A_{n}$ to build a $\uparrow$-DFTA $D_{n}$ leads to consider states which are subsets of $Q$. The state language of a state $\left\{q_{k_{1}} \ldots q_{k_{n}}\right\}$ is a composite residual language. It is the union of $t_{q_{k_{1}}}^{-1} L \ldots t_{q_{k_{n}}}^{-1} L$.

In canonical $\uparrow$-RFTAs, all state languages are prime residual languages.

Theorem 2. Let $L$ be a regular tree language and let us consider the $\uparrow-F T A$ $A_{\text {can }}=\left(Q, \mathcal{F}, Q_{f}, \Delta\right)$ defined by:

- $Q$ is in bijection with the set of all prime bottom-up residual languages of $L$. We denote by $t_{q}$ a ground term such that $q$ is associated with $t_{q}^{-1} L$ in this bijection

$-Q_{f}$ is the set of all elements $q$ of $Q$ such that $t_{q}^{-1} L$ contains the void context $\diamond$

- $\Delta$ contains all the rules $f\left(q_{1}, \ldots, q_{n}\right) \rightarrow q$ such that $t_{q}^{-1} L \subseteq\left(f\left(t_{q_{1}}, \ldots, t_{q_{n}}\right)\right)^{-1} L$ and all the rules $a \rightarrow q$ such that $a \in \mathcal{F}_{0}$ and $t_{q}^{-1} L \subseteq a^{-1} L$.

$A_{\text {can }}$ is a $\uparrow-R F T A$, it is the smallest $\uparrow-R F T A$ in number of states which recognizes $L$, and it is unique up to a renaming of its states.

Sketch of proof There are three things to prove in this theorem: the canonical $\uparrow$-RFTA $A_{\text {can }}=\left(Q, \mathcal{F}, Q_{f}, \Delta\right)$ of a regular tree language $L$ recognizes $L$, it is a $\uparrow$-RFTA, and there cannot be any strictly smaller $\uparrow$-RFTA which recognizes $L$. The three points are proved in this order.

We first have to prove the equality $L\left(A_{\text {can }}\right)=L$. It follows from the identity $(\circledast) \forall t, t^{-1} L=\bigcup_{q \in Q, t \rightarrow{ }_{A c a n}^{*} q} t_{q}^{-1} L$ which can be proved inductively on the height of $t$. Using this property, we have:

$t \in L \Leftrightarrow \diamond \in t^{-1} L \underset{\circledast}{\Leftrightarrow} \diamond \in \bigcup_{q \in Q, t \rightarrow{ }_{A_{c a n}}^{*} q} t_{q}^{-1} L \Leftrightarrow \exists q_{f} \in Q_{f}, t \rightarrow{ }_{A_{c a n}}^{*} q_{f} \Leftrightarrow t \in L\left(A_{c a n}\right)$

The equality between $L$ and $L\left(A_{\text {can }}\right)$ helps us to prove the characterization of $\uparrow$-RFTA: $t_{q}^{-1} L=C_{q}^{A_{\text {can }}}$ where $C_{q}^{A_{\text {can }}}$ is the state language of $q$ in $A_{\text {can }}$.

The last point can be proved in such a way. In a $\uparrow$-RFTA, any residual language is a union of state languages, and any state language is a residual language. So any prime residual language is a state language, so there is at least as much states in a $\uparrow$-RFTA as prime residual languages admitted by its corresponding tree language. 
The canonical automaton is uniquely defined determined by the tree language under consideration, but there may be other automata which have the same number of states. The canonical $\uparrow$-RFTA is unique because it has the maximum number of rules. Even though all its states are associated to prime residual languages, the automaton considered in the proof of Proposition 1 is not the canonical one because some rules are missing: $\bigcup_{k=1}^{n}\left\{f\left(q_{k}, q_{0}\right) \rightarrow q_{k-1}, f\left(q_{0}, q_{k}\right) \rightarrow q_{k-1}\right\}$ and $\bigcup_{q \in Q}\left\{f\left(q, q_{0}\right) \rightarrow q_{*}, f\left(q, q_{0}\right) \rightarrow q_{*}\right\}$.

\section{Top-Down residual finite tree automata}

The definition of top-down residual finite tree automata $(\downarrow-R F T A)$ is tightly correlated with the definition of $\uparrow$-RFTA. Similarly to $\uparrow$-RFTA, $\downarrow$-RFTA are defined as non-deterministic tree automata where each state language is a residual language. Any $\downarrow$-RFTA can be transformed in a canonical equivalent $\downarrow$-RFTA - minimal in the number of states and unique up to state renaming.

The main difference between the bottom-up and the top-down case is in the problem of the expressive power of tree automata. The three classes of bottom-up tree automata, $\uparrow$-DFTA, $\uparrow$-RFTA or $\uparrow$-FTA, have the same expressive power. In the top-down case, deterministic, residual and non-deterministic tree automata have different expressive power. This makes the canonical form of $\downarrow$-RFTA more interesting. Compared to the minimal form of $\downarrow$-DFTA, it can be smaller when both exist, and it exists for a wider class of tree languages.

Let us introduce $\downarrow$-RFTA through their similarity with $\uparrow$-RFTA, then study this specific problem of expressiveness.

\subsection{Analogy with bottom-up residual tree automata}

Let us formally define state languages in the top-down case:

Definition 3. Let $L$ be a regular tree language over a ranked alphabet $\mathcal{F}$, let $A$ be a top-down tree automaton which recognizes $L$, and let $q$ be a state of this automaton. The state language of $L$ relative to $q$, written $L_{q}$, is the set of terms which are accepted by $q$ :

$$
L_{q}=\left\{t \in \mathcal{T}(\mathcal{F}) \mid q(t) \rightarrow_{A}^{*} t\right\} .
$$

It follows from this definition some properties similar to those already studied in the previous section. Firstly, state languages are generally not residual languages. Secondly, residual languages are unions of state languages. Let us define $Q_{c}$ :

$$
Q_{c}=\left\{q \mid q \in Q, \exists q_{i} \in I, q_{i}(c[\diamond]) \rightarrow_{A}^{*} c[q(\diamond)]\right\} .
$$

We have the following relation between state languages and residual languages.

Lemma 1. Let $L$ be a tree language and let $A=(Q, \mathcal{F}, I, \Delta)$ be a top-down tree automaton which recognizes $L$. Then $\forall c \in \mathcal{C}(\mathcal{F}), \bigcup_{q \in Q_{c}} L_{q}=c^{-1} L$. 
These similarities lead us to this definition of top-down residual tree automata:

Definition 4. A top-down Residual Finite Tree Automaton ( $\downarrow-R F T A)$ recognizing a tree language $L$ is a $\downarrow-F T A A=(Q, \mathcal{F}, I, \Delta)$ such that: $\forall q \in Q, \exists c \in \mathcal{C}(\mathcal{F})$, $L_{q}=c^{-1} L$.

Languages defined in the proof of Proposition 1 are still interesting here to define examples of top-down residual tree automata:

Example 3. Let us consider again the family of tree languages $L_{n}$, and the family of corresponding $\uparrow$-RFTA $A_{n}$. For every $n$, let $A_{n}^{\prime}$ be the $\downarrow$-RFTA defined by: $Q=\left\{q_{*}, q_{0}, \ldots, q_{n}\right\}, Q_{i}=\left\{q_{0}\right\}$ and $\Delta=\left\{q_{*}(a) \rightarrow a, q_{n}(a) \rightarrow a, q_{*}(f) \rightarrow\right.$ $\left.f\left(q_{*}, q_{*}\right)\right\} \cup \bigcup_{k=1}^{n}\left\{q_{k-1}(f) \rightarrow f\left(q_{k}, q_{*}\right), q_{k-1}(f) \rightarrow f\left(q_{*}, q_{k}\right)\right\}$.

For every $k \leq n$, the state language of $q_{k}$ is equal to $L_{n-k}$. And, $L_{n-k}$ is the top-down residual language of $c_{k}$, where $c_{k}$ is a context whose height from the root to the special constant $\diamond$ is $k$ and $c_{k}$ does not contain any path whose length is smaller or equal to $n$. The state language of $q_{*}$ is $\mathcal{T}(\mathcal{F})$. And, $\mathcal{T}(\mathcal{F})$ is the top-down residual language of $L_{n}$ relative to $c_{*}$, where $c_{*}$ is a context who contains a path whose length is $n$. So $A_{n}^{\prime}$ is a $\downarrow$-RFTA. Moreover, it is easy to verify that $A_{n}^{\prime}$ recognizes $L_{n}$.

\subsection{The expressive power of top-down tree automata}

Top-down deterministic automata and path-closed languages A tree language $L$ is path-closed if:

$$
\forall c \in C(\mathcal{F}), c\left[f\left(t_{1}, t_{2}\right)\right] \in L \wedge c\left[f\left(t_{1}^{\prime}, t_{2}^{\prime}\right)\right] \in L \Rightarrow c\left[f\left(t_{1}, t_{2}^{\prime}\right)\right] \in L
$$

The reader should note that the definition only considers binary symbols, the definition can easily be extended to $n$-ary symbols. The class of languages that $\downarrow$-DFTA can recognize is the class of path-closed languages Vir81].

Context-deterministic automata and homogeneous languages. Podelski and Nivat in NP97 have defined l-r-deterministic top-down tree automata. In the present paper, let us call them top-down context-deterministic tree automata.

Definition 5. A top-down context-deterministic tree automaton ( $\downarrow$-CFTA) $A$ is $a \downarrow-F T A$ such that for every context $c \in \mathcal{C}(\mathcal{F}), Q_{c}$ is either the empty set or a singleton set.

An homogeneous language is a tree language $L$ satisfying:

$$
\forall c \in C(\mathcal{F}), c\left[f\left(t_{1}, t_{2}\right)\right] \in L \wedge c\left[f\left(t_{1}, t_{2}^{\prime}\right)\right] \in L \wedge c\left[f\left(t_{1}^{\prime}, t_{2}\right)\right] \Rightarrow c\left[f\left(t_{1}^{\prime}, t_{2}^{\prime}\right)\right] \in L
$$

Again, the definition can easily be extended from the binary case to $n$-ary symbols. They have shown that the class of languages recognized by $\downarrow$-CFTA is the class of homogeneous languages. 
The hierarchy A $\downarrow$-DFTA is a $\downarrow$-CFTA. For $\downarrow$-CFTA and $\downarrow$-RFTA, we have the following result:

Lemma 2. Any trimmed $\downarrow-C F T A$ is a $\downarrow$-RFTA.

Proof. Let $A=(Q, \mathcal{F}, I, \Delta)$ be a trimmed $\downarrow$-CFTA recognizing a tree language $L$. As $A$ is trimmed, all states are reachable, so for every $q$, there exists a $c$ such that $q \in Q_{c}$. Then, by definition of a $\downarrow$-CFTA, for every $q$, there exists a $c$ such that $\{q\}=Q_{c}$. Using Lemma 1, we have:

$$
\forall q \in Q, \exists c \in \mathcal{C}(\mathcal{F}), L_{q}=c^{-1} L .
$$

stating that $A$ is a $\downarrow$-RFTA.

Therefore, if we denote by $\mathcal{L}_{\mathcal{C}}$ the class of tree languages recognized by a class of automata $\mathcal{C}$, we obtain the following hierarchy:

$$
\mathcal{L}_{\downarrow-D F T A} \subseteq \mathcal{L}_{\downarrow-C F T A} \subseteq \mathcal{L}_{\downarrow-R F T A} \subseteq \mathcal{L}_{\downarrow-F T A}
$$

The hierarchy is strict

- Let $L=\{f(a, b), f(b, a)\} . L_{1}$ is homogeneous but not path-closed. Therefore $L$ can be recognized by a $\downarrow$-CFTA, but can not be recognized by a $\downarrow$-DFTA.

- The tree languages $L_{n}$ in the proof of Proposition 11 are not recognized by $\downarrow$-CFTA. We can easily verify that $L_{n}$ is not homogeneous. Indeed, if $t$ is a term which has a path whose length is equal to $n-1$, and $t^{\prime}$ a term which does not have any path whose length is smaller than $n, f(t, t), f\left(t, t^{\prime}\right), f\left(t^{\prime}, t\right)$ belong to $L_{n}$, but $f\left(t^{\prime}, t^{\prime}\right)$ does not. And, we have already shown that $L_{n}$ is recognized by a $\downarrow$-RFTA.

- Let $L^{\prime}=\{f(a, b), f(a, c), f(b, a), f(b, c), f(c, a), f(c, b)\} . L^{\prime}$ is a finite language, therefore it is a regular tree language which can be recognized by a $\downarrow$-FTA. $L^{\prime}$ cannot be recognized by a $\downarrow$-RFTA. To prove that, let us consider $A^{\prime}$ a $\downarrow$-FTA which recognizes $L^{\prime}$. The top-down residual languages of $L^{\prime}$ are $\{a, b\},\{a, c\},\{b, c\}$ and $L^{\prime}$. As $A^{\prime}$ recognizes $L^{\prime}$, it recognizes $f(a, b)$. This implies the existence of three states $q_{1}, q_{2}, q_{3}$ and three rules $q_{1}(f) \rightarrow f\left(q_{2}, q_{3}\right)$, $q_{2}(a) \rightarrow a$, and $q_{3}(b) \rightarrow b$. If $A^{\prime}$ was a $\downarrow$-RFTA, then $q_{2}$ would accept a residual language. As $q_{2}$ accepts $a$, it would accept either $\{a, b\}$ or $\{a, c\}$. Similarly, $q_{3}$ would accept either $\{a, b\}$ or $\{b, c\}$. In these conditions, and thanks to the rule $q_{1}(f) \rightarrow f\left(q_{2}, q_{3}\right), A^{\prime}$ would recognize $f(a, a), f(b, b)$ or $f(c, c)$. So $A^{\prime}$ cannot be a $\downarrow$-RFTA.

Therefore, we obtain the following result:

Theorem 3. $\mathcal{L}_{\downarrow-D F T A} \subsetneq \mathcal{L}_{\downarrow-C F T A} \subsetneq \mathcal{L}_{\downarrow-R F T A} \subsetneq \mathcal{L}_{\downarrow-F T A}$

So top-down residual tree automata are strictly more expressive than contextdeterministic tree automata. But as far as we know, there is no straightforward characterization of the tree languages recognized by $\downarrow$-RFTA. 


\subsection{The canonical form of top-down residual tree automata}

The problem of the canonical form of top-down tree automata is similar to the bottom-up case. Whereas there is no way to reduce a non-deterministic top-down tree automaton to a unique canonical form, a top-down residual tree automaton can take such a form. Its definition is similar to the definition of the canonical bottom-up tree automaton.

In the same way that we have defined composite bottom-up residual language, a top-down residual language of $L$ is composite if and only if it is the union of the top-down residual languages that it strictly contains and a residual language is prime if and only if it is not composite.

Theorem 4. Let $L$ be a tree language in the class $\mathcal{L}_{\downarrow-R F T A}$. Let us consider the $\downarrow$-RFTA $A_{\text {can }}=(Q, \mathcal{F}, I, \Delta)$ defined by:

- $Q$ is a set of state in bijection with the prime residual languages of L. For each of these residual languages, there exists a $c_{q}$ such that $q$ is associated with $c_{q}^{-1} L$ in this bijection.

- I is the set of prime residuals which are subsets of $L$.

- $\Delta$ contains all the rules $q(a) \rightarrow a$ such that $a$ is a constant and $c_{q}[a] \in L$, and all the rules $q(f) \rightarrow f\left(q_{1}, \ldots, q_{n}\right)$ such that for all $t_{1} \ldots t_{n}$ where $t_{i} \in c_{q_{i}}^{-1} L$, $c_{q}\left[f\left(t_{1}, \ldots, t_{n}\right)\right] \in L$.

$A_{\text {can }}$ is a $\downarrow-R F T A$, it is the smallest $\downarrow-R F T A$ in number of states which recognizes $L$, and it is unique up to a renaming of its states.

\section{Sketch of proof}

The proof is mainly based on this lemma: $t \in c_{q}^{-1} L \Leftrightarrow t \in L_{q}^{A_{\text {can }}}$

where $L_{q}^{A_{\text {can }}}$ is the state language of $q$ in the automaton $A_{\text {can }}$.

This lemma is proved by induction on the height of $t$. This is not a straightforward induction. It involves the rules of a $\downarrow$-RFTA automaton $A^{\prime}$ which recognizes $L$. Its existence is granted by the hypothesis of the theorem.

Once this is proved, it can be easily deduced that $A_{\text {can }}$ recognizes $L$ and is a RFTA. As there is one state per prime residual in $A_{c a n}$, it is minimal in number of states.

\section{$5 \quad$ Decidability issues}

Some decision problems naturally arise with the definition of RFTA. Most of these problems are solved just noting that one can build all residual languages of a given regular language $L$ defined by a non-deterministic tree automaton. In the bottom-up case, the state languages of the minimal $\uparrow$-RFTA which recognizes $L$ are exactly the residual languages of $L$, and this automaton can be built with the subset construction. In the top-down case, the subset construction does not necessarily gives us an automaton which recognizes exactly $L$, but it gives us the set of all residual languages. Therefore, knowing whether a tree automaton is a RFTA, whether a residual language is prime or composite, and whether a tree automaton is a canonical RFTA are decidable. These problems have not been deeply studied in terms of complexity, but they are at least as hard as the similar problems with strings, that is they are PSPACE-hard (DLT02a). 


\section{Conclusion}

We have defined new classes of non-deterministic tree automata. In the bottomup case, we get another characterization of regular tree languages. More interestingly, in the top-down case, we obtain a subclass of the regular tree languages. For both cases, we have a canonical form and the size of residual tree automata can be much smaller than equivalent (when exist) deterministic ones.

We are currently extending these results to the case of unranked trees because our application domain is concerned with html and xml documents. Also, we are designing learning algorithms for residual finite tree automata extending previous algorithms for residual finite string automata [DLT01, DLT02b].

\section{References}

$\mathrm{CDG}^{+}$97. H. Comon, M. Dauchet, R. Gilleron, F. Jacquemard, D. Lugiez, S. Tison, and M. Tommasi. Tree automata techniques and applications. Available on: http://www.grappa.univ-lille3.fr/tata, 1997.

DLT01. F. Denis, A. Lemay, and A. Terlutte. Learning regular languages using rfsa. In ALT 2001, number 2225 in Lecture Notes in Artificial Intelligence, pages 348-363. Springer Verlag, 2001.

DLT02a. F. Denis, A. Lemay, and A. Terlutte. Residual finite state automata. Fundamenta Informaticae, 51(4):339-368, 2002.

DLT02b. F. Denis, A. Lemay, and A. Terlutte. Some language classes identifiable in the limit from positive data. In ICGI 2002, number 2484 in Lecture Notes in Artificial Intelligence, pages 63-76. Springer Verlag, 2002.

Fer02. Henning Fernau. Learning tree languages from text. In Proc. 15th Annual Conference on Computational Learning Theory, COLT 2002, pages 153 168, 2002.

GK02. Sally A. Goldman and Stephen S. Kwek. On learning unions of pattern languages and tree patterns in the mistake bound model. Theoretical Computer Science, 288(2):237 - 254, 2002.

Gol67. E.M. Gold. Language identification in the limit. Inform. Control, 10:447474, 1967.

GS84. F. Gcseg and M. Steinby. Tree Automata. Akademiai Kiado, 1984.

GS96. F. Gcseg and M. Steinby. Tree languages. In G. Rozenberg and A. Salomaa, editors, Handbook of Formal Languages, volume 3, pages 1-68. Springer Verlag, 1996.

LPH00. Ling Liu, Calton Pu, and Wei Han. XWRAP: An XML-enabled wrapper construction system for web information sources. In ICDE, pages 611-621, 2000 .

MLM01. M. Murata, D. Lee, and M. Mani. "Taxonomy of XML Schema Languages using Formal Language Theory". In Extreme Markup Languages, Montreal, Canada, 2001.

Nev02. F. Neven. Automata theory for xml researchers. SIGMOD Rec., 31(3):3946, 2002.

NP97. M. Nivat and A. Podelski. Minimal ascending and descending tree automata. SIAM Journal on Computing, 26(1):39-58, February 1997.

Sak90. Y. Sakakibara. Learning context-free grammars from structural data in polynomial time. Theoretical Computer Science, 76:223 - 242, 1990.

Tha73. J.W. Thatcher. Tree automata: an informal survey. In A.V. Aho, editor, Currents in the theory of computing, pages 143-178. Prentice Hall, 1973. 
Tho84. Wolfgang Thomas. Logical aspects in the study of tree languages. In Proceedings of the 9th International Colloquium on Trees in Algebra and Programming, CAAP '84, pages 31 - 50, 1984.

Vir81. J. Viragh. Deterministic ascending tree automata. Acta Cybernetica, 5:3342, 1981. 


\section{A Appendix}

\section{A.1 Proof of Equation (1)}

Let $L$ be a tree language and $\left(Q, \mathcal{F}, Q_{f}, \Delta\right)$ a $\uparrow$-FTA which recognizes it. We show that $\forall t \in T(\mathcal{F}), t^{-1} L=\bigcup_{t \rightarrow{ }_{A}^{*} q} C_{q}$.

Let $t \in \mathcal{T}(\mathcal{F})$, and $c \in t^{-1} L . c[t] \in L$, so there exists $q_{f} \in Q_{f}$ and $q \in Q$ such that $c[t] \rightarrow_{A}^{*} c[q] \rightarrow_{A}^{*} q_{f}$, where $t \rightarrow_{A}^{*} q$ and $c \in C_{q}$. So $c \in \bigcup_{t \rightarrow{ }_{A}^{*} q} C_{q}$. So $t^{-1} L \subseteq \bigcup_{t \rightarrow{ }_{A}^{*} q} C_{q}$

Let $t \in \mathcal{T}(\mathcal{F})$, and $c \in \bigcup_{t \rightarrow{ }_{A}^{*} q} C_{q}$. There exists a $q \in Q$ such that $t \rightarrow{ }_{A}^{*} q$ and $c \in C_{q}$. So there exists $q_{f} \in Q_{f}$ such that $c[t] \rightarrow_{A}^{*} c[q] \rightarrow_{A}^{*} q_{f}$. So $c \in t^{-1} L$. So $\bigcup_{t \rightarrow{ }_{A}^{*} q} C_{q} \subseteq t^{-1} L$

\section{A.2 Proof of the theorem 2}

Theorem 5. The canonical $\uparrow-R F T A$ recognizing a regular tree language is the smallest $\uparrow-R F T A$ which recognizes it. Therefore, $\uparrow-R F T A$ accepts a unique and minimal representation.

The first point we have to demonstrate in this theorem is that the canonical $\uparrow$-RFTA that we have defined recognizes $L$.

Before this demonstration, we need to establish two properties of residual languages:

Lemma 3. Let $L$ a regular language.

$$
\forall i, 1 \leq i \leq n, t_{i}^{-1} L \subseteq t_{i}^{\prime-1} L \Rightarrow f\left(t_{1}, \ldots, t_{n}\right)^{-1} L \subseteq f\left(t_{1}^{\prime}, \ldots, t_{n}^{\prime}\right)^{-1} L
$$

Proof. This lemma can be proven inductively on $i$. Let $t_{1} \ldots t_{n}$ such that for all $i, t_{i}^{-1} L$ is a subset of $t_{i}^{\prime-1} L$. Let $c$ in $f\left(t_{1}, \ldots, t_{n}\right)^{-1} L$. Let us assume that $c\left[f\left(t_{1}^{\prime}, \ldots, t_{i-1}^{\prime}, t_{i}, \ldots, t_{n}\right)\right] \in L$. This implies that $c\left[f\left(t_{1}^{\prime}, \ldots, t_{i-1}^{\prime}, \diamond, t_{i+1}, \ldots, t_{n}\right)\right] \in$ $t_{i}^{-1} L$, and therefore $c\left[f\left(t_{1}^{\prime}, \ldots, t_{i-1}^{\prime}, \diamond, t_{i+1}, \ldots, t_{n}\right)\right] \in t_{i}^{\prime-1} L$.

So $c\left[f\left(t_{1}^{\prime}, \ldots, t_{i}^{\prime}, t_{i+1}, \ldots, t_{n}\right)\right] \in L$. Inductively, $c\left[f\left(t_{1}^{\prime}, \ldots, t_{n}^{\prime}\right)\right] \in L$.

So $f\left(t_{1}, \ldots, t_{n}\right)^{-1} L \subseteq f\left(t_{1}^{\prime}, \ldots, t_{n}^{\prime}\right)^{-1} L$.

\section{Lemma 4.}

$$
\forall i, 1 \leq i \leq n, t_{i}^{-1} L=\bigcup_{j_{i}} t_{i, j_{i}}^{-1} L \Rightarrow f\left(t_{1}, \ldots, t_{n}\right)^{-1} L=\bigcup_{j_{1} \ldots j_{n}} f\left(t_{1, j_{1}}, \ldots, t_{n, j_{n}}\right)^{-1} L
$$

Here, $\bigcup_{j_{1} \ldots j_{n}}$ has to be understood as 'the union of all the possible combination of $j_{1} \ldots j_{n}$

Proof. Let $t_{1} \ldots t_{n}$ and for all $i \leq n, t_{i, 1} \ldots t_{i, m_{i}}$ such that $t_{i}^{-1} L=\bigcup_{1 \leq j_{i} \leq m_{i}} t_{i, j_{i}}^{-1} L$.

$$
\begin{gathered}
\forall t_{1, j_{1}} \ldots t_{n, j_{n}}, \forall i \leq n, t_{i, j_{i}}^{-1} L \subseteq t_{i}^{-1} L \Rightarrow_{\text {lemma }} \\
\forall t_{1, j_{1}} \ldots t_{n, j_{n}}, f\left(t_{1, j_{1}} \ldots t_{n, j_{n}}\right)^{-1} L \subseteq f\left(t_{1}, \ldots, t_{n}\right)^{-1} L \Rightarrow
\end{gathered}
$$




$$
\bigcup_{j_{1} \ldots j_{n}} f\left(t_{1, j_{1}}, \ldots, t_{n, j_{n}}\right)^{-1} L \subseteq f\left(t_{1}, \ldots, t_{n}\right)^{-1} L
$$

Now, let $c$ in $f\left(t_{1}, \ldots, t_{n}\right)^{-1} L$.

$$
c\left[f\left(t_{1}, \ldots, t_{n}\right)\right] \in L \Rightarrow c\left[f\left(\diamond, t_{2}, \ldots, t_{n}\right)\right] \in t_{1}^{-1} L
$$

As $t_{1}^{-1} L=\bigcup t_{1, j}^{-1} L$, there exists $t_{1, m_{1}}$ such that $c\left[f\left(\diamond, t_{2}, \ldots, t_{n}\right)\right] \in t_{1, m_{1}}^{-1} L$. So $c\left[f\left(t_{1, m_{1}}, t_{2}, \ldots, t_{n}\right)\right] \in L$.

It can be proven inductively on $i$ that there exists $t_{1, m_{1}} \ldots t_{n, m_{n}}$ such that $c\left[f\left(t_{1, k_{1}}, \ldots, t_{n, m_{n}}\right)\right] \in L$. So $c \in f\left(t_{1, m_{1}}, \ldots, t_{n, m_{n}}\right)^{-1} L$. So:

$$
f\left(t_{1}, \ldots, t_{n}\right)^{-1} L \subseteq \bigcup_{j_{1} \ldots j_{n}} f\left(t_{1, j_{1}}, \ldots, t_{n, j_{n}}\right)^{-1} L
$$

Now, we can prove inductively this lemma, which is the main step to prove the equality between $L$ and $L\left(A_{c a n}\right)$

\section{Lemma 5.}

$$
\forall t, t^{-1} L=\bigcup_{t \rightarrow A_{\text {can }}^{*} q} t_{q}^{-1} L
$$

Proof. Let us prove this lemma inductively. Let $h(t)$ be the height of $t$.

Let us assume that $h(t)=1$, so $t=a$ where $a$ is a constant. A residual is a union of prime residuals, so:

$$
a^{-1} L=\bigcup_{t_{q}^{-1} L \subseteq a^{-1} L} t_{q}^{-1} L
$$

As $t_{q}^{-1} L \subseteq a^{-1} L$ if and only if $A_{\text {can }}$ contains the rule $a \rightarrow q$ :

$$
a^{-1} L=\bigcup_{a \rightarrow A_{\text {can }}^{*}} t_{q}^{-1} L
$$

Now let us assume that for any term $t$ such that $h(t) \leq k$, lemma 5 is true.

Let $t=f\left(t_{1}, \ldots, t_{n}\right)$ such that $h(t)=k+1$.

$$
\begin{gathered}
h(t)=k+1 \Rightarrow \forall i \leq n, h\left(t_{i}\right)=k \Rightarrow \forall i, t_{i}^{-1} L=\bigcup_{t_{i} \rightarrow A_{c a n}^{*} q_{i, j_{i}}} t_{q_{i, j_{i}}}^{-1} L \Rightarrow_{\text {lemma日 }} \\
t^{-1} L=\bigcup_{t_{i} \rightarrow A_{A_{c a n}}^{*} q_{i, j_{i}}} f\left(t_{1, q_{1}}, \ldots, t_{n, q_{n}}\right)^{-1} L
\end{gathered}
$$

Any residual is a union of prime residuals, so for all $j_{1} \ldots j_{n}$ :

$$
f\left(t_{q_{1, j_{1}}}, \ldots, t_{q_{n, j_{n}}}\right)^{-1} L=\bigcup_{t_{q}^{-1} L \subseteq f\left(t_{q_{1, j_{1}}}, \ldots, t_{q_{n, j_{n}}}\right)^{-1} L} t_{q}^{-1} L
$$

So:

$$
t^{-1} L=\bigcup_{t_{i} \rightarrow{ }_{A_{c a n}}^{*} q_{i, j_{i}}} f\left(t_{1, q_{1}}, \ldots, t_{n, q_{n}}\right)^{-1} L \Rightarrow
$$




$$
t^{-1} L=\bigcup_{t_{i} \rightarrow{ }_{A_{c a n}}^{*} q_{i, j_{i}}}\left(\underset{t_{q}^{-1} L \subseteq f\left(t_{q_{1, j_{1}}}, \ldots, t_{q_{n, j_{n}}}\right)^{-1} L}{\bigcup} t_{q}^{-1} L\right)
$$

As $t_{q}^{-1} L \subseteq f\left(t_{q_{1, j_{1}}}, \ldots, t_{q_{n, j_{n}}}\right)^{-1} L$ if and only if $A_{c a n}$ contains the rule $f\left(q_{1, j_{1}}, \ldots, q_{n, j_{n}}\right) \rightarrow q$

$$
t^{-1} L=\bigcup_{t \rightarrow A_{\text {can }}^{*} q} t_{q}^{-1} L
$$

The equality between $L$ and $L\left(A_{c a n}\right)$ is formalized as such:

Lemma 6. The canonical $\uparrow-R F T A \mathcal{A}_{\text {can }}$ of a language $L$ recognizes $L$, that is:

$$
\exists q_{f} \in Q_{f}, t \rightarrow{ }_{A_{c a n}}^{*} q_{f} \Leftrightarrow t \in L
$$

Proof. Let $t \in \mathcal{T}(\mathcal{F})$ and $q_{f} \in Q_{f}$ such that $t \rightarrow_{A_{c a n}}^{*} q_{f}$.

$$
t \rightarrow{ }_{A_{\text {can }}}^{*} q_{f} \Rightarrow t_{q_{f}}^{-1} L \subset \bigcup_{t \rightarrow A_{A_{c a n}}^{*} q_{j}} t_{q_{j}}^{-1} L \Rightarrow_{\text {lemma }} t_{q_{f}}^{-1} L \subset t^{-1} L
$$

As $\diamond \in t_{q_{f}}^{-1} L, \diamond \in t^{-1} L$, so $t \in L$.

Let $t \in L$.

$$
\begin{gathered}
\diamond \in t^{-1} L \Rightarrow \exists q_{j} \mid t \rightarrow{ }_{A_{\text {can }}}^{*} q_{j} \wedge \diamond \in t_{q_{j}}^{-1} L \Rightarrow \\
\exists q_{j} \mid t \rightarrow_{A_{\text {can }}} q_{j} \wedge q_{j} \in Q_{f}
\end{gathered}
$$

Now, we have to prove that the canonical $\uparrow$-RFTA is a $\uparrow$-RFTA. In order to do this, we need to establish this lemma:

Lemma 7. Let $t_{q}^{-1} L$ and $t_{q^{\prime}}^{-1} L$ be prime bottom-up residual languages of $L$. Let $C_{q}^{A_{\text {can }}}$ and $C_{q^{\prime}}^{A_{\text {can }}}$ be sets of contexts accepted by $q$ and $q^{\prime}$ in then canonical automaton of $L A_{\text {can }}$. Then:

$$
t_{q^{\prime}}^{-1} L \subset t_{q}^{-1} L \Rightarrow C_{q^{\prime}}^{A_{\text {can }}} \subset C_{q}^{A_{\text {can }}}
$$

Proof. Let $t_{q}$ and $t_{q^{\prime}}$ such that $t_{q^{\prime}}^{-1} L \subset t_{q}^{-1} L$. For all $t_{q_{1}} \ldots t_{q_{n}}, f\left(t_{q_{1}}, \ldots, t_{q^{\prime}}, \ldots, t_{q_{n}}\right)^{-1} L \subset$ $f\left(t_{q_{1}}, \ldots, t_{q}, \ldots, t_{q_{n}}\right)$ (lemma 3).

The construction of the set of rules of the canonical automaton implies that:

$$
f\left(q_{1}, \ldots, q_{n}\right) \rightarrow q^{\prime} \in \Delta \Leftrightarrow t_{q^{\prime}}^{-1} L \subseteq f\left(t_{q_{1}}, \ldots, t_{q_{n}}\right)^{-1} L
$$

So:

$$
\begin{gathered}
f\left(q_{1}, \ldots, q^{\prime}, \ldots, q_{n}\right) \rightarrow q^{\prime \prime} \in \Delta \Rightarrow \\
t_{q^{\prime \prime}}^{-1} L \in f\left(t_{q_{1}}, \ldots, t_{q^{\prime}}, \ldots, t_{q_{n}}\right)^{-1} L \Rightarrow \\
t_{q^{\prime \prime}}^{-1} L \in f\left(t_{q_{1}}, \ldots, t_{q}, \ldots, t_{q_{n}}\right)^{-1} L \Rightarrow \\
\quad f\left(q_{1}, \ldots, q, \ldots, q_{n}\right) \rightarrow q^{\prime \prime} \in \Delta
\end{gathered}
$$

So each context accepted by $q^{\prime}$ is accepted by $q$.

So $C_{q^{\prime}}^{A_{\text {can }}} \subset C_{q}^{A_{c a n} \text {. }}$ 
Lemma 8. The canonical RFTA $A_{\text {can }}$ of a language $L$ is a residual finite tree automata.

Proof. Let $t_{q}^{-1} L$ be a prime residual language of $L$. Thanks to lemma 5 :

$$
t_{q}^{-1} L=\bigcup_{t_{q} \rightarrow{ }_{A_{c a n}}^{*} q^{\prime}} t_{q^{\prime}}^{-1} L
$$

If $t_{q}^{-1} L$ would strictly contain all the $t_{q^{\prime}}^{-1} L$ of the union, it would be composite. As it is prime, $t_{q}^{-1} L$ is itself an element of this union, so $t_{q} \rightarrow_{A_{c a n}}^{*} q$.

Equation (11) tells us that:

$$
t_{q}^{-1} L=\bigcup_{t_{q} \rightarrow_{A c a n}^{*} q^{\prime}} C_{q^{\prime}}
$$

So $C_{q} \subset t_{q}^{-1} L$.

For all $q^{\prime}$ such that $t_{q} \rightarrow_{A_{c a n}}^{*} q^{\prime}, t_{q^{\prime}}^{-1} L \subset t_{q}^{-1} L$, so $C_{q^{\prime}} \subset C_{q}$ (lemma $\mathbb{0}$ ). As the union of all $C_{q^{\prime}}$ is equal to $t_{q}^{-1} L, t_{q}^{-1} L \subset C_{q}$

So $t_{q}^{-1} L=C_{q}$, so every prime residual language is accepted by its corresponding state.

So $A_{c a n}$ is a RFTA.

Lemma 9. The canonical RFTA $A_{\text {can }}$ of a language $L$ is the smallest RFTA which recognizes $L$.

Proof. Let $A_{\text {can }}$ be the canonical RFTA of a language $L$, and $t$ such that $\nexists q \in$ $Q, t^{-1} L=C_{q}$.

Thanks to lemma 旬, $t^{-1} L=\bigcup_{t \rightarrow{ }_{A c a n}^{*}} q t_{q}^{-1} L$. As $\nexists q \in Q, t^{-1} L=t_{q}^{-1} L, t^{-1} L$ is a union of residuals that it strictly contains. So $t^{-1} L$ is a composite residual.

So for all prime residuals $t^{-1} L$, there is a $q$ such that $t^{-1} L=C_{q} . A_{\text {can }}$ contains as much states as prime residuals in $L$, so it is the smallest RFTA which recognizes $L$.

\section{A.3 Proof of the theorem 4}

Theorem 6. Let $L$ be a language recognized by a $\downarrow-R F T A$. The canonical topdown residual tree automaton of $L$ is the smallest $\downarrow-R F T A$ which recognizes $L$.

In order to prove this theorem, let us firstly prove these lemma:

Lemma 10. Let $A=(Q, \mathcal{F}, I, \Delta)$ be a $\downarrow$-RFTA which recognizes $L$. For any prime residual $c^{-1} L$, there exists a state $q \in Q$ such that $L_{q}=c^{-1} L$.

Proof. Let $c$ be a context of $L$ such that $\nexists q \in Q, c^{-1} L=L_{q}$.

Lemma 1 implies that $c^{-1} L=\bigcup_{q \in Q_{c}} L_{q}$ and none of these $L_{q}$ are equal to $c^{-1} L$. As $\forall q \in Q, L_{q}=c_{q}^{-1} L$, we have $c^{-1} L=\bigcup_{q \in Q_{c}} c_{q}^{-1} L$ where none of the $c_{q}^{-1} L$ are equal to $c^{-1} L$. So $c^{-1} L$ is composite. 
Now, let us make the main part of the demonstration: let us prove that each prime residual language is exactly accepted by a state of the canonical $\downarrow$-RFTA.

Lemma 11. Let $L$ be a language recognized by a $\downarrow-R F T A$. Let $A_{\text {can }}=(Q, \mathcal{F}, I, \Delta)$ be its canonical automaton. For all $q$ in $Q, c_{q}^{-1} L=L_{q}$.

Proof. As seen in the definition, $Q$ is in bijection with the set of all residual languages, so for all $q$ there exists a corresponding $c_{q}^{-1} L$. Let us prove inductively on the height of $t$ that $t \in c_{q}^{-1} L \Leftrightarrow t \in L_{A_{c a n}, q}$. Let us call $H(n)$ this hypothesis when $h(t) \leq n$.

Firstly, let us prove $H(1)$.

Let $t$ such that $h(t)=1$ and $t \in c_{q}^{-1} L$. As $h(t)=1, t=a$ where $a$ is a constant. As $t \in c_{q}^{-1} L, c_{q}[a] \in L$. So $\Delta$ contains the rule $q(a) \rightarrow a$, so $t \in L_{A_{\text {can }}, q}$. Reciprocally, $t \in L_{A_{\text {can }}, q}$ where $t=a$ implies that $\Delta$ contains the rule $q(a) \rightarrow a$. This rule exists in the canonical automata if and only if $a$ is a constant and $c_{q}[a] \in L$. So $c_{q}[a] \in L$, so $t \in c_{q}^{-1} L$.

Now, let us assume that $H(l)$ is true when $l<k$. Let us prove that $H(k)$ is true.

Let $t=f\left(t_{1}, \ldots, t_{n}\right) \in c_{q}^{-1} L$ such that $h(t)=k$. For all $t_{i}$ where $1 \leq i \leq n$, $t_{i} \in c_{q}\left[f\left(t_{1}, \ldots, t_{i-1}, \diamond, t_{i+1}, \ldots, t_{n}\right)\right]^{-1} L$.

Now, let us consider $A^{\prime}=\left(Q^{\prime}, \mathcal{F}, I^{\prime}, \Delta^{\prime}\right)$ a $\downarrow$-RFTA which recognizes $L$. As $L$ is recognized by a $\downarrow$-RFTA, $A^{\prime}$ exists. We will use this automaton to prove the existence of a rule $q \rightarrow f\left(q_{1}, \ldots, q_{n}\right)$ such that for all $i, q_{i}\left[t_{i}\right] \rightarrow_{A}^{*} t_{i}$ in $A_{\text {can }}$.

As $c_{q}^{-1} L$ is prime, there exists a $q^{\prime} \in Q^{\prime}$ such that $L_{A^{\prime}, q^{\prime}}=c_{q}^{-1} L$ (lemma 10). As $t \in c_{q}^{-1} L$, there exists in $\Delta^{\prime}$ a rule $q^{\prime} \rightarrow f\left(q_{1}^{\prime}, \ldots, q_{n}^{\prime}\right)$ such that for all $i$, $1 \leq i \leq n$, we have $t_{i} \in L_{A^{\prime}, q_{i}^{\prime}}$.

For all $t_{1}^{\prime} \ldots t_{n}^{\prime}$ such that $t_{i}^{\prime} \in L_{A^{\prime}, q_{i}^{\prime}}, f\left(t_{1}^{\prime}, \ldots, t_{n}^{\prime}\right) \in c_{q}^{-1} L$.

As $L_{A^{\prime}, q_{i}^{\prime}}$ is a residual, it is either a prime residual or a composite residual. If it is a prime residual, there exists a $q_{i} \in Q$ such that $L_{A^{\prime}, q_{i}^{\prime}}=c_{q_{i}}^{-1} L$ and $t_{i} \in c_{q_{i}}^{-1} L$. If it is a composite residual, there exists a $q_{i} \in Q$ such that $c_{q_{i}}^{-1} L \subset L_{A^{\prime}, q_{i}^{\prime}}$ and $t_{i} \in c_{q_{i}}^{-1} L$.

So there exists $q_{1} \ldots q_{n}$ such that $t_{i} \in c_{q_{i}}^{-1} L \subset L_{A^{\prime}, q_{i}^{\prime}}$. So for all $t_{1}^{\prime} \ldots t_{n}^{\prime}$ in $c_{q_{1}}^{-1} L \ldots c_{q_{n}}^{-1} L, f\left(t_{1}^{\prime}, \ldots, t_{n}^{\prime}\right) \in L_{A^{\prime}, q^{\prime}}^{-1} L=c_{q}^{-1} L$. So the rule $q(f) \rightarrow f\left(q_{1}, \ldots, q_{n}\right)$ exists in $\Delta$.

For all $t_{i}, h\left(t_{i}\right)<k$, so as we have assumed that $H(l)$ is right when $l<k$, $H\left(h\left(t_{i}\right)\right)$ is right. So for all $i, t_{i} \in L_{A_{\text {can }}, q_{i}}$. As $q(f) \rightarrow f\left(q_{1}, \ldots, q_{n}\right), t \in L_{A_{\text {can }}, q}$.

We have proven that $t \in c_{q}^{-1} L \Rightarrow t \in L_{A_{c a n}, q}$. Now let us prove that $t \in L_{A_{\text {can }}, q} \Rightarrow t \in c_{q}^{-1} L$.

Let $t=f\left(t_{1}, \ldots, t_{n}\right) \in L_{A_{\text {can }}, q}$ such that $h(t)=k$.

There exist $q_{1} \ldots q_{t}$ such that $q\left(f\left(t_{1} \ldots t_{n}\right)\right) \rightarrow_{A}^{*} f\left(q_{1}\left(t_{1}\right), \ldots, q_{n}\left(t_{n}\right)\right) \rightarrow_{A}^{*} f\left(t_{1}, \ldots, t_{n}\right)$. For all $i, t_{i} \in L_{A_{\text {can }}, q_{i}}$ and $h\left(t_{i}\right)<k$, so $H\left(h\left(t_{i}\right)\right)$ is assumed to be true, so $t_{i} \in c_{q_{i}}^{-1} L$. The existence of the rule $q(f) \rightarrow f\left(q_{1}, \ldots, q_{n}\right)$ in $\Delta$ implies that for all $t_{1}^{\prime} \ldots t_{n}^{\prime}$ such that $t_{i}^{\prime} \in c_{q_{i}}^{-1} L, c_{q}\left[f\left(t_{1}^{\prime}, \ldots, t_{n}^{\prime}\right)\right] \in L$. So $t \in c_{q}^{-1} L$.

So $\mathrm{H}(\mathrm{k})$ is true. We have proven inductively that for any $t, t \in L_{A_{\text {can }}, q} \Leftrightarrow$ $t \in c_{q}^{-1} L$. 
Lemma 12. $A_{\text {can }}=<Q, \mathcal{F}, Q_{i}, \Delta>$ is a $\downarrow$-RFTA, recognizes $L$, and is minimal in number of states.

Proof. Let us prove that lemma 11 implies that $L\left(A_{\text {can }}\right)=L$. Let $t \in L . \diamond^{-1} L=$ $L$ is a residual, so it is a union of prime residuals. So there exists $q_{i} \in Q$ such that $t \in c_{q_{i}}^{-1} L$ and $c_{q_{i}}^{-1} L \subseteq L$. As $c_{q_{i}}^{-1} L=L_{A_{c a n}, q_{i}}$, we have $t \in L_{A_{c a n}, q_{i}} \cdot c_{q_{i}}^{-1} L \subseteq L$, so $q_{i}$ is initial, so $t \in L\left(A_{\text {can }}\right)$.

Reciprocally, let $t \in L\left(A_{\text {can }}\right)$. There exists a $q_{i} \in I$ such that $t \in L_{A_{c a n}, q_{i}}$. $c_{q_{i}}^{-1} L=L_{q_{i}}$, so $t \in c_{q_{i}}^{-1} L$. As $q_{i}$ is initial, $c_{q_{i}}^{-1} L$ is a subset of $L$. So $t \in L$. So $L=L\left(A_{\text {can }}\right)$.

So $A_{\text {can }}$ recognizes $L$. For any $q, L_{q}=c_{q}^{-1} L$, so $A_{\text {can }}$ is a RFTA. For any prime residual of $L$, there exists a state in the RFTA which recognizes it. As there are one state per prime residual in $A_{c a n}, A_{c a n}$ is minimal in number of states. 